\title{
Associação de adjuvantes com herbicidas na dessecação e no controle em pós- emergência de plantas daninhas na cultura do trigo ${ }^{1}$
}

\author{
Adjuvant association with herbicides in burndown and post-emergency control of \\ weeds in wheat crop \\ Cleber Daniel de Goes Maciel ${ }^{2}$, David Willians Moraes ${ }^{3}$, Marcelo Gonçalves Balan ${ }^{3}$
}

\begin{abstract}
Resumo - Este trabalho teve com objetivo avaliar associações de adjuvantes aos herbicidas utilizados na dessecação e no controle em pós-emergência de plantas daninhas na cultura do trigo. O delineamento utilizado foi de blocos casualizados com quatro repetições, em esquema fatorial 2 x $5+1$, constituído por duas formas de dessecação de pré-semeadura, representadas por aplicação única e em sequencial, com cinco combinações de adjuvantes (sem adjuvante; Triunfo $515^{\circledR}$; Grap Super Gun ${ }^{\circledR}$; Assist ${ }^{\circledR}$ e Fulltec ${ }^{\circledR}$ ) e uma testemunha sem aplicação. Todos os tratamentos efetuados em dessecação foram submetidos ao complemento de controle em pósemergência com metsulfuron-methyl ou metsulfuron-methyl + 2,4-D, juntamente com os adjuvantes dos respectivos tratamentos de dessecação. Os adjuvantes Triunfo $515^{\circledR}$, Grap Super Gun $^{\circledR}$ e Assist $^{\circledR}\left(70 \mathrm{~mL} \mathrm{ha}^{-1}\right)$ promoveram incremento no controle de capim-amargoso (Digitaria insularis) para as formulações de glyphosate Polaris ${ }^{\circledR}$ e Atanor $480^{\circledR}\left(2,25 \mathrm{~L} \mathrm{ha}^{-1}\right)$, em aplicação única de pré-semeadura. Para trapoeraba (Commelina benghalensis), apesar da baixa eficiência da dessecação de pré-semeadura, a utilização dos adjuvantes incrementou a ação do glyphosate isoladas e/ou em mistura com 2,4-D e 2,4-D + metsulfuron-methyl. As associações entre metsulfuron-methyl $+2,4-\mathrm{D}$, com os adjuvantes, controlaram satisfatoriamente a trapoeraba em pós-emergência. Metsulfuron-methyl $\left(2,4 \mathrm{~g} \mathrm{ha}^{-1}\right)$ e metsulfuron-methyl + 2,4-D $\left(2,4+322,4 \mathrm{~g} \mathrm{ha}^{-}\right.$ ${ }^{1}$ ), associados aos adjuvantes estudados não promoveram ação fitotóxica prejudicial à cultura do trigo cv BRS 220.
\end{abstract}

Palavras-chave: Digitaria insularis, Commelina benghalensis, Triticum aestivum.

Abstract - This work aimed to evaluate associations between adjuvant to herbicides used in burndown and weeds control in post emergence conditions in wheat crop. The experiment was conducted by using randomized complete blocks with four repetitions, in factorial scheme 2 x $5+$ 1 , constituted by two burndown forms in pre sowing, represented by only one application and in sequence, with five adjuvant combinations (without adjuvant; Triunfo 515 ${ }^{\circledR}$; Grap Super Gun ${ }^{\circledR}$; Assist $^{\circledR}$ and Fulltec ${ }^{\circledR}$ ) and a check without application. All treatments developed in burndown were submitted to the complement of post emergence control with metsulfuron-methyl or

\footnotetext{
${ }^{1}$ Recebido para publicação em 22/06/2011 e na forma revisada em 10/11/2011.

2 Docente, Universidade Estadual de Maringá - Campus Avançado de Umuarama/CAU. End. Estrada da Paca s/ número - Bairro São Cristovão CEP 87507-190, Umuarama-Paraná. 〈cdgmaciel2@uem.br〉

3 Aluno do Programa de Aperfeiçoamento/Especialização em Proteção de Plantas, Faculdade Integrado de Campo Mourão, CEI, 87300-970. Campo Mourão/PR.
} 
metsulfuron-methyl $+2.4-\mathrm{D}$, join to adjuvant from the respective burndown treatments. Triunfo $515^{\circledR}$, Grap Super Gun ${ }^{\circledR}$ and Assist ${ }^{\circledR}\left(70 \mathrm{~mL} \mathrm{ha}^{-1}\right)$ adjuvant provided increased in Digitaria insularis control for glyphosate Polaris ${ }^{\circledR}$ and Atanor $480^{\circledR}\left(2.25 \mathrm{~L} \mathrm{ha}^{-1}\right)$ formulations, in unique application in pre sowing. For Commelina benghalensis, besides burndown reduction efficiency in pre sowing conditions, the use of adjuvant increased glyphosate action isolated and/or in mixture with 2.4-D and 2.4-D + metsulfuron-methyl. Associations between metsulfuron-methyl + 2.4-D, with the adjuvant, controlled satisfactory $C$. benghalensis in post emergency. Metsulfuronmethyl $\left(2.4 \mathrm{~g} \mathrm{ha}^{-1}\right)$ and metsulfuron-methyl $+2.4-\mathrm{D}\left(2.4+322.4 \mathrm{~g} \mathrm{ha}^{-1}\right)$, associated to the studied adjuvant did not cause harmful plant toxic action to wheat crop BRS 220 genotype.

Key-words: Digitaria insularis, Commelina benghalensis, Triticum aestivum.

\section{Introdução}

O trigo (Triticum aestivum L.) além de ocupar o primeiro lugar em volume de produção mundial entre os cereais caracterizase no Brasil, com uma das mais importantes culturas na estação fria, por ser uma das alternativas na sucessão com soja em sistema de semeadura direta. Atualmente, a produção anual brasileira de trigo oscila entre 5 a 6 milhões de toneladas (CONAB, 2010), e o consumo tem se mantido em torno de 10 milhões de toneladas, havendo constante necessidade de importação deste cereal (Comissão Sul-Brasileira de Pesquisa de Trigo, 2005).

O trigo tem importante papel econômico e nutricional na alimentação humana, uma vez que é largamente utilizado na indústria alimentícia (Ferreira, 2003; Gieco et al., 2004). Nesse sentido, a qualidade de sua farinha é resultado da interação das condições de cultivo (solo, clima, pragas e cultivar), somado à interferência das plantas daninhas, operações de colheita, secagem e armazenamento (El-Dashi \& Miranda, 2002; Gutkoski \& Neto, 2002; Roman et al., 2006).

Com relação ao manejo de plantas daninhas na cultura do trigo, Ambrosi et al. (2000) mencionaram que em apenas 76,8\%, $75,1 \%$ e $45,2 \%$, respectivamente, das lavouras nos Estados do Paraná, Santa Catarina e Rio Grande do Sul, apresentaram medidas de controle adequadas. Em termos mundiais, estima-se que a quebra da produção potencial de trigo devido à interferência das plantas daninhas não exceda $10 \%$, estando o prejuízo causado pelas infestantes, relacionado à agressividade de cada espécie (IAPAR, 2002).

\section{Velloso \& Dal' Piaz} constataram em trigo, cevada e centeio redução de produtividade pela convivência durante todo o ciclo com populações infestantes de azevém (Lolium multiflorum L.) e gorga (Spergula arvensis L.), da ordem de 12,4 \%; 32,6 \% e $18,5 \%$, respectivamente. Agostinetto et al. (2008) determinaram que as medidas de controle das plantas daninhas em trigo devem ser adotadas no período entre 12 e 24 dias após a emergência da cultura.

Entre os herbicidas mais utilizados para o controle de plantas daninhas no trigo, tanto na dessecação como em pós-emergência, encontram-se 2,4-D e metsulfuron-methyl (Vargas \& Roman, 2005). Albrecht et al. (2010) mencionaram que devido ao baixo custo do metsulfuron-methyl, juntamente com o surgimento de plantas daninhas com resistência a este produto, consideráveis abusos estão ocorrendo, do ponto de vista da dose ou frequência de uso, que podem causar danos fitointoxicação à cultura do trigo. Assim, a combinação de altas dosagens e uso de maneira inadequada, juntamente às condições ambientais desfavoráveis podem afetar 
negativamente o desenvolvimento e o potencial produtivo do trigo (Salvagiotti et al., 2001).

Desta forma, o trabalho teve como objetivo avaliar associações de adjuvantes aos herbicidas nas operações de dessecação e controle em pós-emergência de plantas daninhas na cultura do trigo.

\section{Material e Métodos}

O trabalho foi desenvolvido em condições de campo no município de Campo Mourão/PR, durante o período de maio a setembro/2010. O solo da área experimental foi classificado como LATOSSOLO VERMELHO, textura média-arenosa, com $25 \%$ de argila, $69 \%$ de areia e $6 \%$ de silte.

O sistema de preparo do solo empregado foi o de semeadura direta sobre palhada de soja. A adubação de semeadura foi realizada utilizando-se $250 \mathrm{~kg} \mathrm{ha}^{-1}$ do formulado 8-20-20 (N-P-K) e cobertura com 60 $\mathrm{kg} \mathrm{ha}^{-1}$ de uréia 25 dias após a semeadura. Para os demais tratos culturais, incluindo manejo de pragas e doenças, foram utilizadas as recomendações técnicas da Comissão SulBrasileira de Pesquisa de Trigo (2005).

A semeadura da cultivar de trigo BRS 220 foi realizada em 22/05/2010 utilizando-se espaçamento de $17 \mathrm{~cm}$ entre linhas, na profundidade de $3 \mathrm{~cm}$ e densidade de 70 sementes por metro. As parcelas foram representadas por 20 linhas de 10 metros de comprimento $\left(40 \mathrm{~m}^{2}\right)$, sendo utilizado como área útil apenas $27 \mathrm{~m}^{2}$ constituído pelas fileiras centrais da unidade experimental, desprezando $0,5 \mathrm{~m}$ das bordaduras.

O delineamento experimental utilizado foi $\mathrm{o}$ de blocos completos casualizados com quatro repetições, em esquema fatorial 2 × $5+1$, constituído por duas formas de dessecação com misturas em tanque representadas por aplicação única e sequencial (dividida em duas aplicações), com cinco combinações de adjuvantes (sem adjuvante; Triunfo 515 ${ }^{\circledR}$; Grap Super Gun ${ }^{\circledR}$; Assist $^{\circledR}$ e Fulltec $^{\circledR}$ ) e testemunha sem aplicação. Todos os tratamentos efetuados em dessecação foram submetidos ao complemento de controle em pós-emergência com metsulfuron-methyl e metsulfuron-methyl $+2,4-\mathrm{D}$, juntamente com os mesmos adjuvantes dos respectivos tratamentos utilizados na dessecação. Os herbicidas, com suas respectivas dosagens, encontram-se demonstrados na Tabela 1.

As aplicações dos herbicidas foram realizadas utilizando-se pulverizador costal pressurizado por $\mathrm{CO}_{2}$, equipado com barra de quatro pontas jato plano duplo AD/D 110-02, do fabricante Magno ${ }^{\circledR}$, espaçadas de $50 \mathrm{~cm} \mathrm{e}$ com consumo de calda de $200 \mathrm{~L} \mathrm{ha}^{-1}$.

No momento das aplicações o solo encontrava-se com umidade e ausência de ventos; sendo em média, a temperatura e umidade relativa do ar nas operações de dessecação e pós-emergência da ordem de $20^{\circ} \mathrm{C}$ e $65 \%$ e $23^{\circ} \mathrm{C}$ e $60 \%$, respectivamente. $\mathrm{Na}$ Tabela 2, encontram-se representados os dados meteorológicos referentes às médias diárias de temperatura $\left({ }^{\circ} \mathrm{C}\right)$ e pluviometria $(\mathrm{mm})$.

As principais plantas daninhas presentes na área experimental, no momento da aplicação de dessecação, foram, em média, representadas por: 6 plantas $\mathrm{m}^{-2}$ de capimamargoso (Digitaria insularis) e 15 plantas $\mathrm{m}^{-2}$ de trapoeraba (Commelina benghalensis), ambas em estágio reprodutivo. Na aplicação em pós-emergência, a comunidade infestante foi constituída principalmente por: trapoeraba (28 plantas $\mathrm{m}^{-2}$ ), com plantas de 2 a 5 folhas e com mais de 5 a 7 ramificações, caracterizadas por plantas remanescentes da baixa eficácia de controle da operação de dessecação de présemeadura.

Tabela 1. Tratamentos herbicidas utilizados em manejo de dessecação da infestação e no controle em pós-emergência das plantas daninhas na cultura do trigo. Campo Mourão/PR, 2010. 


\begin{tabular}{|c|c|c|}
\hline \multicolumn{2}{|c|}{ Dessecação de pré-semeadura } & \multirow{2}{*}{$\begin{array}{l}\text { Controle em pós-emergência } \\
\text { (28 DDS) }\end{array}$} \\
\hline Aplicação única (40 DAS) & Aplicação sequencial (25 DAS) & \\
\hline 1. glyphosate Polaris ${ }^{\circledR / 1}$ & glyphosate Agripec ${ }^{\circledR / 3}+2,4-\mathrm{D}^{14}$ & metsulfuron-methyl $^{/ 6}$ \\
\hline 2. glyp. Polaris ${ }^{\circledR / 1}+$ Triunfo $515^{\circledR / 8}$ & glyp. Agripec ${ }^{\circledR / 3}+2,4-\mathrm{D}^{/ 4}+$ Triunfo $515^{\circledR / 7}$ & 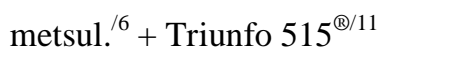 \\
\hline 3. glyp. Polaris ${ }^{\circledR / 1}+$ Grap S. Gun ${ }^{\circledR / 9}$ & $\begin{array}{l}\text { glyp. Agripec }{ }^{\circledR / 3}+2,4-D^{14}+\text { Grap Super } \\
\text { Gun }^{\circledR / 8}\end{array}$ & metsul. ${ }^{16}+$ Grap Super Gun ${ }^{\circledR / 12}$ \\
\hline 4. glyp. Polaris ${ }^{\circledR / 1}+$ Assist $^{\circledR / 10}$ & glyp. Agripec ${ }^{\circledR / 3}+2,4-D^{14}+$ Assist $^{\circledR / 9}$ & metsul. $^{16}+$ Assist $^{\circledR / 13}$ \\
\hline 5. glyp. Polaris ${ }^{\circledR / 1}+$ Fulltec $^{\circledR / 11}$ & glyp. Agripec ${ }^{\circledR / 3}+2,4-D^{14}+$ Fulltec $^{\circledR / 10}$ & metsul. $^{/ 6}+$ Fulltec $^{\circledR / 14}$ \\
\hline 6. glyphosate Atanor ${ }^{\circledR / 2}$ & $\begin{array}{l}\text { glyp. Agripec }{ }^{\circledR / 3}+2,4-D^{/ 4}+\text { metsulfuron- } \\
\text { methyl }^{/ 6}\end{array}$ & metsul. ${ }^{16}+2,4-\mathrm{D}^{15}$ \\
\hline 7. glyp. Atanor ${ }^{\circledR / 2}+$ Triunfo $515^{\circledR / 8}$ & $\begin{array}{l}\text { glyp. Agripec }{ }^{\circledR / 3}+2,4-\mathrm{D}^{/ 4}+\text { metsul. }^{/ 6}+ \\
\text { Triunfo515 }\end{array}$ & $\begin{array}{l}\text { metsul. }_{515^{\circledR / 11}} \\
\end{array}$ \\
\hline 8. glyp. Atanor ${ }^{\circledR / 2}+$ Grap S. Gun ${ }^{\circledR / 9}$ & $\begin{array}{l}\text { glyp. Agripec }{ }^{\circledR / 3}+2,4-D^{14}+\text { metsul. }^{16}+ \\
\text { Grap S. Gun }^{\circledR / 8}\end{array}$ & $\operatorname{metsul.}_{\text {Gun }^{\circledR / 12}}{ }^{16}+2,4-\mathrm{D}^{15}+$ Grap S. \\
\hline 9. glyp. Atanor ${ }^{\circledR / 2}+$ Assist $^{\circledR / 10}$ & $\begin{array}{l}\text { glyp. Agripec }{ }^{\circledR / 3}+2,4-\mathrm{D}^{/ 4}+\text { metsul. }^{16}+ \\
\text { Assist }^{\circledR / 9}\end{array}$ & metsul. ${ }^{/ 6}+2,4-\mathrm{D}^{15}+$ Assist $^{\circledR / 13}$ \\
\hline 10. glyp. Atanor ${ }^{\circledR / 2}+$ Fulltec $^{\circledR / 11}$ & $\begin{array}{l}\text { glyp. Agripec }{ }^{\circledR / 3}+2,4-\mathrm{D}^{/ 4}+\text { metsul. }^{/ 6}+ \\
\text { Fulltec }^{\circledR / 10}\end{array}$ & metsul. ${ }^{16}+2,4-\mathrm{D}^{15}+$ Fulltec $^{\circledR / 14}$ \\
\hline testemunha sem aplicação & - & - \\
\hline $\begin{array}{l}\text { Obs. - DAS = dias antes da semeac } \\
\text { Glifosato Atanor } 480{ }^{\circledR} / 3=\text { Glifosato } \\
\left.\mathrm{c} \mathrm{ha}^{-1} \text { ou } 322,4 \text { g e.a. } \text { ha }^{-1}\right) ;{ }^{16}=\text { Ally } \\
. / 11 / 12 / 14=80 \mathrm{mI}\end{array}$ & $\begin{array}{l}\text { - DDS = dias após a semeadura; doses } \\
\text { ripec } 480^{\circledR}\left(2,25 \mathrm{~L} \mathrm{pc} \mathrm{ha}^{-1} \text { ou } 1080 \mathrm{~g} \text { e.a. }\right. \\
\left.0 \mathrm{~g} \mathrm{pc} \mathrm{ha}^{-1} \text { ou } 2,4 \mathrm{~g} \mathrm{e.a.} \mathrm{ha}{ }^{-1}\right) \text {; dosagens do } \\
\mathrm{pc} \mathrm{ha}^{-1}\end{array}$ & $\begin{array}{l}\text { s herbicidas: }{ }^{11}=\text { Polaris }^{\circledR},{ }^{12}= \\
\left.{ }^{-1}\right) ;{ }^{/ 4 / 5}=\text { DMA } 806 \mathrm{BR}^{\circledR}(0,4 \mathrm{~L} \\
\text { adjuvantes: }{ }^{/ 7 / 8 / 10}=70 \mathrm{~mL} \mathrm{pc} \mathrm{ha}^{-}\end{array}$ \\
\hline
\end{tabular}

As avaliações foram porcentagem de controle das plantas daninhas em aplicação única aos 7, 14 DAA (dias após aplicação), e seqüencial de 21/7 e 28/14 DAA para avaliação da dessecação de manejo pré-semeadura, e aos 7, 14 e 21 DAA em pós-emergência, onde a partir da testemunha sem aplicação, se considerou satisfatório o controle igual ou superior a $80 \%$. A fitointoxicação da cultura do trigo foi avaliada aos 7, 14, 21/7 e 28/14 DAA, por meio de notas visuais onde $0 \%$ corresponde à ausência de injúrias e 100\% à morte das plantas (SBCPD, 1995). Foi realizada a colheita da área útil de cada parcela para determinar a influência dos tratamentos sobre a produtividade de grãos $\left(\mathrm{kg} \mathrm{ha}^{-1}\right)$.

Os dados obtidos foram submetidos à análise de variância pelo teste $\mathrm{F}$ e as médias comparadas pelo teste de Tukey, em nível de 5 $\%$ de probabilidade.

Tabela 2. Dados meteorológicos, referentes à precipitação e temperatura média mensal ocorrida durante o desenvolvimento do experimento. Campo Mourão/PR, 2010. 


\begin{tabular}{|c|c|c|c|c|c|c|c|c|c|c|c|c|}
\hline $\begin{array}{c}\text { Dias/ } \\
\text { mês }\end{array}$ & Abr & Mai & Jun & Jul & Ago & Set & Abr & Mai & Jun & Jul & Ago & Set \\
\hline 1 & & & & & & & 24 & 20 & 14 & 22 & 22 & 23 \\
\hline 2 & & & & & & & 24 & 20 & 15 & 21 & 21 & 25 \\
\hline 3 & 1 & & & & 16 & & 23 & 20 & 16 & 20 & 20 & 24 \\
\hline 4 & 1 & & & & & & 23 & 21 & 17 & 19 & 19 & 25 \\
\hline 5 & & & 21 & & 22 & & 24 & 18 & 14 & 21 & 21 & 15 \\
\hline 6 & 10 & & & & & & 18 & 18 & 12 & 21 & 21 & 16 \\
\hline 7 & & & & & & 5 & 18 & 21 & 12 & 20 & 20 & 17 \\
\hline 8 & & 15 & & & & 8 & 18 & 21 & 14 & 19 & 19 & 18 \\
\hline 9 & & 12 & & & & & 19 & 16 & 17 & 20 & 20 & 19 \\
\hline 10 & & & & & & & 19 & 16 & 16 & 22 & 22 & 20 \\
\hline 11 & & & & & & & 20 & 16 & 16 & 21 & 21 & 21 \\
\hline 12 & & 8 & & & & & 21 & 12 & 16 & 21 & 21 & 24 \\
\hline 13 & $*$ & & & 10 & & $* * * * *$ & 22 & 13 & 15 & 19 & 19 & 26 \\
\hline 14 & & & & & & 3 & 22 & 14 & 15 & 17 & 17 & 26 \\
\hline 15 & & & & & & & 22 & 14 & 18 & 18 & 18 & 13 \\
\hline 16 & & & & 30 & & & 22 & 19 & 19 & 16 & 16 & 19 \\
\hline 17 & & & & 8 & & & 23 & 19 & 20 & 13 & 13 & 19 \\
\hline 18 & & 78 & & & & & 24 & 17 & 21 & 14 & 14 & 25 \\
\hline 19 & & 17 & $* * * *$ & & & & 23 & 13 & 17 & 18 & 18 & 20 \\
\hline 20 & & & & & & & 23 & 11 & 20 & 16 & 16 & 17 \\
\hline 21 & & & & & & & 25 & 15 & 22 & 17 & 17 & 23 \\
\hline 22 & & $* * *$ & 8 & & & & 26 & 17 & 23 & 21 & 21 & 26 \\
\hline 23 & 80 & & & & & & 26 & 19 & 19 & 20 & 20 & 27 \\
\hline 24 & 52 & & & 5 & & 6 & 19 & 20 & 21 & 19 & 19 & 20 \\
\hline 25 & & 8 & & & & & 21 & 17 & 21 & 21 & 21 & 18 \\
\hline 26 & & & & & & 38 & 23 & 17 & 22 & 20 & 20 & 21 \\
\hline 27 & 16 & & & & & 25 & 22 & 18 & 21 & 15 & 15 & 18 \\
\hline 28 & $* *$ & & & & & 16 & 20 & 18 & 20 & 18 & 18 & 19 \\
\hline 29 & 7 & & & & & & 20 & 17 & 21 & 15 & 15 & 18 \\
\hline 30 & & & & & & & 22 & 19 & 19 & 17 & 17 & 18 \\
\hline 31 & & 12 & & & & & & 14 & & 19 & 19 & \\
\hline$\sum(\mathbf{m m})$ & $\mathbf{1 6 7}$ & $\mathbf{1 5 0}$ & $\mathbf{2 9}$ & $\mathbf{5 3}$ & $\mathbf{3 8}$ & $\mathbf{1 0 1}$ & - & - & - & - & - & - \\
\hline
\end{tabular}

* $1^{\mathrm{a}}$ aplicação de dessecação; ** $2^{\mathrm{a}}$ aplicação de dessecação; *** Semeadura; **** Aplicação em pós-emergência;

\section{Resultados e Discussão}

Para dessecação de pré-semeadura do capim-amargoso (D. insularis) pode-se observar que aos 7 e 14 DAA o controle foi ineficiente $(\leq 41,3 \%)$ e significativamente semelhante para aplicação única de glyphosate $\left(1080\right.$ g e.a. ha $\left.{ }^{-1}\right)$, nas formulações comerciais Polaris ${ }^{\circledR}$ e Glifosato Atanor $480^{\circledR}$, independentemente da associação dos adjuvantes estudados (Tabela 3). A partir dos 21 DAA única e 7 DAA sequencial (21/7 DAA), observou-se que o nível de controle da referida espécie foi no mínimo satisfatório $(\geq$
$86,3 \%$ ) para todos os tratamentos que receberam aplicação sequencial, superando a condição de aplicação única nesse período, a qual exceto para aplicação única de Glifosato Atanor $480^{\circledR}+$ Grap Super Gun ${ }^{\circledR}$, onde não foi caracterizada diferenças significativas da associação Glifosato Agripec $480^{\circledR}+2,4-D+$ metsulfuron-methyl + Grap Super Gun ${ }^{\circledR}$. 
Tabela 3. Porcentagem de controle na dessecação de capim-amargoso (Digitaria insularis) aos 7, 14, 21/7 e 28/14 DAA, utilizando apenas uma única aplicação (1 aplicação) ou aplicação sequencial (2 aplicações). Campo Mourão/PR, 2010.

\begin{tabular}{|c|c|c|c|c|c|c|}
\hline \multirow{2}{*}{$\begin{array}{c}\text { TRATAMENTOS } \\
\text { Aplicação única (40 DAS) / } \\
\text { Aplicação sequencial (25 DAS) }\end{array}$} & \multirow{2}{*}{$\frac{7 \text { DAA }}{\text { Única }}$} & 14 DAA & \multicolumn{2}{|c|}{ 21/7 DAA } & \multicolumn{2}{|c|}{ 28/14 DAA } \\
\hline & & Única & Única & Sequencial & Única & Sequencial \\
\hline $\begin{array}{l}\text { 1. glyph. Polaris }{ }^{\circledR / 1} / / \\
\text { glyph. Agripec }{ }^{\circledR / 3}+2,4-\mathrm{D}^{14}\end{array}$ & $17,5 \quad b$ & $32,5 \quad \mathrm{a}$ & $59,0 \quad \mathrm{~d} \quad \mathrm{~B}$ & 88,3 a $A$ & $73,0 \quad b \quad B$ & 100,0 a $A$ \\
\hline $\begin{array}{l}\text { 2. glyph. Polaris }{ }^{\circledR / 1}+\text { Triunfo } 515^{\circledR / 8} / \\
\text { glyph. Agripec }{ }^{\circledR / 3}+2,4-\mathrm{D}^{\prime 4}+\text { Triunfo } 515^{\circledR / 6}\end{array}$ & $18,8 \quad a b$ & $40,0 \quad \mathrm{a}$ & 74,0 abc B & 89,5 a $A$ & 88,3 a $B$ & 100,0 \\
\hline $\begin{array}{l}\text { 3. glyph. Polaris }{ }^{\circledR / 1}+\text { Grap Super Gun }{ }^{\circledR / 9} / \\
\text { glyph. Agripec }{ }^{\circledR / 3}+2,4-D^{/ 4}+\text { Grap Sup Gun }^{\circledR / 7}\end{array}$ & $27,5 \quad a b$ & 41,3 & 75,0 abc B & 90,0 a $A$ & 90,0 a $B$ & 100,0 a $\mathrm{A}$ \\
\hline $\begin{array}{l}\text { 4. glyph. Polaris }{ }^{\circledR / 1}+\text { Assist }^{\circledR / 10} / \\
\text { glyph. Agripec } \\
\end{array}$ & $20,0 \quad a b$ & 37,5 & 68,3 bcd B & $92,8 \quad \mathrm{a} \quad \mathrm{A}$ & $\mathrm{b} \quad \mathrm{B}$ & 100,0 \\
\hline $\begin{array}{l}\text { 5. glyph. Polaris }{ }^{\circledR / 1}+\text { Fulltech }^{\circledR / 11} / \\
\text { glyph. Agripec }{ }^{\circledR / 3}+2,4-\mathrm{D}^{/ 4}+\text { Fulltec }^{\circledR / 9}\end{array}$ & $28,8 \quad \mathrm{a}$ & 37,5 a & 64,5 cd $B$ & 91,5 a $A$ & 74,5 bc B & 100,0 \\
\hline $\begin{array}{l}\text { 6. glyph. Atanor } \\
\text { glyphosate Agripec }^{\circledR / 3}+2,4-\mathrm{D}^{/ 4}+\text { metsulfuron }^{16}\end{array}$ & $23,8 \quad a b$ & 32,5 & 64,0 cd $B$ & 87,5 a $A$ & $75,8 \quad b \quad B$ & 100,0 \\
\hline $\begin{array}{l}\text { 7. glyph. Atanor }{ }^{\circledR / 2}+\text { Triunfo } 515^{\circledR / 8} / \\
\text { glyph. Agripec }{ }^{\circledR / 3}+2,4-\mathrm{D}^{/ 4}+\text { metsul. }^{/ 6}+{\text { Triunfo } 515^{\circledR / 6}}^{\circledR / 2}\end{array}$ & $20,0 \quad a b$ & $38,8 \quad \mathrm{a}$ & 77,8 ab $B$ & 86,3 a $A$ & 87,8 a $\mathrm{B}$ & 100,0 \\
\hline $\begin{array}{l}\text { 8. glyph. Atanor }{ }^{\circledR / 2}+\text { Grap Super Gun }{ }^{\circledR / 9} / \\
\text { glyph. Agripec }{ }^{\circledR / 3}+2,4-D^{/ 4}+\text { metsul }^{1 /}+\text { Grap Sup Gun }{ }^{\circledR / 7}\end{array}$ & $22,5 \quad \mathrm{ab}$ & $37,5 \quad a$ & $84,0 \quad \mathrm{a} \quad \mathrm{A}$ & 90,0 a $A$ & 92,0 a $B$ & 100,0 \\
\hline $\begin{array}{l}\text { 9. glyph. Atanor }{ }^{\circledR / 2}+\text { Assist }^{\circledR / 10} / \\
\text { glyph. Agripec } \\
\end{array}$ & $21,3 \quad a b$ & $36,3 \quad \mathrm{a}$ & 75,3 abc B & 89,5 a $\mathrm{A}$ & 87,5 a $B$ & 100,0 \\
\hline $\begin{array}{l}\text { 10. glyph. Atanor }{ }^{\circledR / 2}+\text { Fulltech }^{\circledR / 11} / \\
\text { glyph. Agripec }{ }^{\circledR / 3}+2,4-D^{/ 4}+\text { metsul. }^{/ 6}+\text { Fulltec }^{\circledR / 9}\end{array}$ & $25,0 \quad a b$ & $35,0 \quad \mathrm{a}$ & $64,5 \mathrm{~cd} B$ & 88,8 a $\mathrm{A}$ & $76,3 \quad b \quad B$ & 100,0 \\
\hline 11. testemunha sem aplicação & $0,0 \quad \mathrm{c}$ & $0,0 \quad b$ & 0,0 e $\mathrm{A}$ & b A & $\mathrm{c} A$ & $\mathrm{~b} \quad \mathrm{~A}$ \\
\hline Teste f (tratamentos) & $11,60 *$ & $28,04 *$ & $\mathrm{CV}(\%$ & $=6,88$ & $\mathrm{CV}(\%$ & $=3,84$ \\
\hline Coeficiente de Variação & 22,19 & 12,90 & Fcal trat $=190,7 *$ & $\mathrm{DMS}_{\text {trat }}=11,81$ & Fcal trat $=605,6^{*}$ & $\mathrm{DMS}_{\text {trat }}=7,52$ \\
\hline DMS $(5 \%)$ & 11,16 & 10,64 & Fcal aplic $=256,2^{*}$ & $\mathrm{DMS}_{\mathrm{Aplic}}=7,07$ & Fcal aplic $=558,4^{*}$ & $\mathrm{DMS}_{\mathrm{Aplic}}=4,50$ \\
\hline
\end{tabular}

Obs. - DAS = dias antes da semeadura; - DDS = dias após a semeadura; Dosagens dos herbicidas: ${ }^{11}=$ Polaris ${ }^{\circledR},{ }^{12}=$ Glifosato Atanor $480{ }^{\circledR / 3}=$ Glifosato Agripec

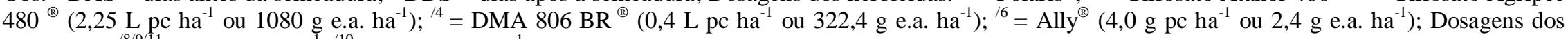
adjuvantes: ${ }^{/ 8 / 9 / 11}=70 \mathrm{~mL} \mathrm{pc} \mathrm{ha}{ }^{-1} ;{ }^{/ 10}=500 \mathrm{~mL} \mathrm{pc} \mathrm{ha}{ }^{-1}$. - Médias seguidas de mesma letra maiúscula na linha e minúscula na coluna não diferem entre si pelo teste Tukey a $5 \%$ de probabilidade. $*=\mathrm{P}<0,05 ;{ }^{\mathrm{NS}}=$ não significativo. 
Tabela 4. Porcentagem de controle na dessecação de trapoeraba (Commelina benghalensis) aos 7, 14, 21/7 e 28/14 DAA, utilizando apenas uma única aplicação (1 aplicação) ou aplicação sequencial (2 aplicações). Campo Mourão/PR, 2010.

\begin{tabular}{|c|c|c|c|c|c|c|}
\hline \multirow{3}{*}{$\begin{array}{c}\text { TRATAMENTOS } \\
\text { Aplicação única (40 DAS) / } \\
\text { Aplicação sequencial (25 DAS) }\end{array}$} & \multirow{2}{*}{$\frac{7 \text { DAA }}{\text { Única }}$} & \multirow{2}{*}{$\frac{14 \text { DAA }}{\text { Única }}$} & \multicolumn{2}{|c|}{$21 / 7 \mathrm{DAA}$} & \multicolumn{2}{|c|}{ 28/14 DAA } \\
\hline & & & Única & Sequencial & Única & Sequencial \\
\hline & $5,0 \quad \mathrm{a}$ & $18,3 \quad b$ & 23,3 b B & $37,5 \quad b \quad A$ & $28,3 \quad c$ & 52,5 ab A \\
\hline $\begin{array}{l}\text { 2. glyph. Polaris }{ }^{\circledR / 1}+\text { Triunfo } 515^{\circledR / 8} / \\
\text { glyph. Agripec }{ }^{\circledR / 3}+2,4-D^{1 / 4}+\text { Triunfo } 515^{\circledR / 6}\end{array}$ & $5,8 \quad \mathrm{a}$ & $32,0 \quad \mathrm{a}$ & $\mathrm{ab} A$ & 50,0 ab $A$ & $51,5 \quad \mathrm{a}$ & 60,0 ab $A$ \\
\hline $\begin{array}{l}\text { 3. glyph. Polaris }{ }^{\circledR / 1}+\text { Grap Super Gun }{ }^{\circledR / 9} / \\
\text { glyph. Agripec }{ }^{\circledR / 3}+2,4-D^{/ 4}+\text { Grap Sup Gun }{ }^{\circledR / 7}\end{array}$ & $5,8 \quad \mathrm{a}$ & $26,3 \quad a b$ & 35,8 ab $B$ & 55,0 a $A$ & 40,8 abc $B$ & a A \\
\hline $\begin{array}{l}\text { 4. glyph. Polaris }{ }^{\circledR / 1}+\text { Assist }^{\circledR / 10} / \\
\text { glyph. Agripec } \\
{ }^{\circledR / 3}+2,4-D^{14}+\text { Assist }^{\circledR / 8}\end{array}$ & 6,5 & $18,5 \quad b$ & $\mathrm{ab} B$ & 45,8 ab $A$ & 40,0 abc $B$ & 55,0 ab $A$ \\
\hline $\begin{array}{l}\text { 5. glyph. Polaris }{ }^{\circledR / 1}+\text { Fulltech }^{\circledR / 11} / \\
\text { glyph. Agripec }{ }^{\circledR / 3}+2,4-\mathrm{D}^{/ 4}+\text { Fulltec }^{\circledR / 9}\end{array}$ & $5,0 \quad \mathrm{a}$ & $23,3 \quad a b$ & 31,5 ab $A$ & 40,8 ab $A$ & 37,0 bc $B$ & 48,8 \\
\hline 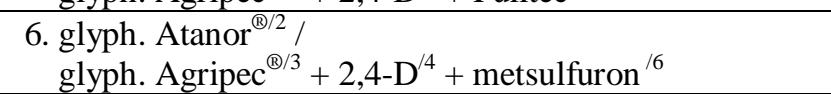 & $5,8 \quad \mathrm{a}$ & $19,0 \quad b$ & $\mathrm{ab} B$ & 40,0 ab A & $30,3 \quad c$ & 49,5 \\
\hline 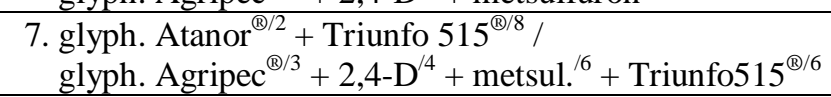 & $5,8 \quad \mathrm{a}$ & $29,5 \quad \mathrm{a}$ & 34,5 ab $A$ & 42,5 ab $A$ & 41,5 abc $B$ & 60,3 ab $A$ \\
\hline $\begin{array}{l}\text { 8. glyph. Atanor }{ }^{\circledR / 2}+\text { Grap Super Gun }{ }^{\circledR / 9} / \\
\text { glyph. Agripec }{ }^{\circledR / 3}+2,4-D^{/ 4}+\text { metsul. }^{/ 6}+\text { Grap Sup Gun } \\
{ }^{\circledR / 7}\end{array}$ & $4,5 \quad \mathrm{a}$ & $30,0 \quad a$ & 34,8 ab $\mathrm{B}$ & 45,3 ab $A$ & 45,3 ab $B$ & 57,0 ab A \\
\hline $\begin{array}{l}\text { 9. glyph. Atanor }{ }^{\circledR / 2}+\text { Assist }^{\circledR / 10} / \\
\text { glyph. Agripec } \\
{ }^{\circledR / 3}+2,4-\mathrm{D}^{/ 4}+\text { metsulf }^{16}+\text { Assist }^{\circledR / 8}\end{array}$ & $7,0 \quad \mathrm{a}$ & $22,8 \quad a b$ & 32,0 ab $B$ & 42,5 ab A & 39,5 abc B & $\mathrm{b} \quad \mathrm{A}$ \\
\hline $\begin{array}{l}\text { 10. glyph. Atanor }{ }^{\circledR / 2}+\text { Fulltech }^{\circledR / 11} / \\
\text { glyph. Agripec }{ }^{\circledR / 3}+2,4-D^{/ 4}+\text { metsul. }^{/ 6}+\text { Fulltec }^{\circledR / 9}\end{array}$ & $7,3 \quad \mathrm{a}$ & $24,5 \quad a b$ & 31,5 ab $B$ & 46,3 ab A & 40,0 abc B & 53,3 ab A \\
\hline 11. testemunha sem aplicação & $0,0 \quad b$ & $0,0 \quad \mathrm{c}$ & c A & $0,0 \quad \mathrm{c} \quad \mathrm{A}$ & $0,0 \quad \mathrm{~d} \quad \mathrm{~A}$ & c A \\
\hline Teste f (tratamentos) & $7,39 *$ & $17,40 *$ & \multicolumn{2}{|c|}{$\mathrm{CV}(\%)=20,33$} & \multicolumn{2}{|c|}{$\mathrm{CV}(\%)=13,38$} \\
\hline Coeficiente de Variação & 27,04 & 18,91 & Fcal trat $=24,5^{*}$ & $\mathrm{DMS}_{\text {trat }}=16,77$ & Fcal trat $=55,5^{*}$ & $\mathrm{DMS}_{\text {trat }}=13,59$ \\
\hline DMS $(5 \%)$ & 3,52 & 10,31 & Fcal aplic $=54,3 *$ & $\mathrm{DMS}_{\text {Aplic }}=10,0$ & Fcal aplic $=137,1^{*}$ & $\mathrm{DMS}_{\mathrm{Aplic}}=8,12$ \\
\hline
\end{tabular}

Obs. - DAS = dias antes da semeadura; - DDS = dias após a semeadura; Dosagens dos herbicidas: ${ }^{11}=$ Polaris $^{\circledR},{ }^{12}=$ Glifosato Atanor $480^{\circledR / 3}=$ Glifosato Agripec

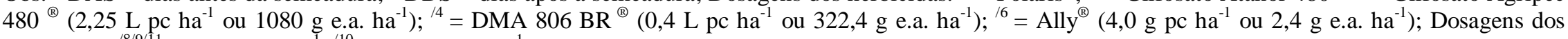
adjuvantes: ${ }^{/ 8 / 9 / 11}=70 \mathrm{~mL} \mathrm{pc} \mathrm{ha}{ }^{-1} ;{ }^{/ 10}=500 \mathrm{~mL} \mathrm{pc} \mathrm{ha}{ }^{-1}$. - Médias seguidas de mesma letra maiúscula na linha e minúscula na coluna não diferem entre si pelo teste Tukey a $5 \%$ de probabilidade. $*=\mathrm{P}<0,05 ;{ }^{\mathrm{NS}}=$ não significativo. . 
Aos 28/14 DAA, os tratamentos da aplicação sequencial atingiram eficácia máxima no controle do capim-amargoso, diferindo significativamente de todas as condições de aplicação única.

As aplicações únicas de Polaris ${ }^{\circledR}+$ Triunfo $515^{\circledR}$, Polaris $^{\circledR}+$ Grap Super Gun ${ }^{\circledR}$, Atanor $480^{\circledR}+$ Triunfo $515^{\circledR}$, Atanor $480^{\circledR}+$ Grap Super Gun ${ }^{\circledR}$ e Atanor $480^{\circledR}+$ Assist $^{\circledR}$, apesar de não terem atingido níveis excelentes $(\geq 95,0 \%)$ de controle de capim-amargoso aos 28 DAA, promoveram controle satisfatório e superior a $86,3 \%$, diferindo significativamente do uso isolado das formulações de glyphosate na presença ou ausência dos adjuvantes até o referido período (Tabela 3). Estes resultados discordam dos obtidos por Dan et al. (2010), por não terem constatado benefícios da adição dos adjuvantes multifuncionais à calda de aplicação de quatro formulações de glyphosate (1080 g e.a. ha ${ }^{-1}$ ), para o controle de capimamargoso (D. insularis) em estádio de desenvolvimento vegetativo. Entretanto, Procópio et al. (2006) ressaltaram-se que para o manejo dessa espécie de planta daninha em estágio de desenvolvimento avançado é importante obter eficiência máxima de controle na operação de dessecação de pré-semeadura, objetivando inviabilizar a ocorrência de rebrotes e/ou re-infestação da mesma após a semeadura da cultura.

Para trapoeraba ( $C$. benghalensis), a dessecação de pré-semeadura apresentou baixa eficácia dos 7 aos 28 DAA $(\leq 51,5 \%)$ para todos os tratamentos em aplicação única, assim como aos 21/7 DAA e 28/14 DAA das aplicações em sequencial $(\leq 65,8 \%)$ (Tabela 4$)$. Entretanto, apesar da baixa eficiência da dessecação da trapoeraba mesmo em aplicação seqüencial, provavelmente ocorrido devido ao uso de dosagem inadequada de glyphosate na primeira aplicação e ao estádio de desenvolvimento avançado da espécie em questão; ressaltamos que de forma geral, a utilização dos adjuvantes incrementou significativamente o desempenho das formulações de glyphosate isoladas e/ou em mistura em tanque com 2,4-D e 2,4-D + metsulfuron-methyl. Esses resultados corroboram com os descritos por Maciel et al. (2011), onde foi constatado que a interação entre o efeito de pontas de pulverização e o uso do adjuvante Triunfo Flex $^{\circledR}\left(0,05 \% \quad \mathrm{v}^{-1}\right)$ promoveram incremento significativo da deposição da calda de pulverização e do controle da trapoeraba (Commelina diffusa) para mistura glyphosate + carfentrazone-ethy $\left(480+8 \mathrm{~g} \mathrm{ha}^{-1}\right)$.

Os dados de porcentagem de controle de trapoeraba em pós-emergência, assim como de fitointoxicação na cultura do trigo estão representados nas Tabelas 5 e 6 . Considerando a avaliação realizada até os 14 DAA, em área submetida à aplicação única ou sequencial da dessecação de pré-semeadura, observou-se que o controle de trapoeraba em pós-emergência da cultura do trigo com metsulfuron-methyl $(2,4 \mathrm{~g}$ e.a. ha $\left.^{-1}\right)$ e metsulfuron-methyl + 2,4-D $(2,4+$ $322,4 \mathrm{~g}$ e.a. $\mathrm{ha}^{-1}$ ) foram ineficientes, mesmo quando associados aos adjuvantes, sendo caracterizados níveis médios de $47 \%$ a $53 \%$ e $63 \%$ a $72 \%$, respectivamente. Não foram constatadas diferenças significativas sobre a eficiência do controle em pós-emergência da trapoeraba até os 21 DAA, entre os sistemas de manejo com aplicação única ou sequencial. Entretanto, os 21 DAA as combinação de metsulfuron-methyl + 2,4-D e adjuvantes, proporcionaram níveis de controle satisfatórios $(\geq 80,0 \%)$ da trapoeraba, diferindo significativamente da aplicação isolada de metsulfuron-methyl, mesmo quando também utilizados em mistura com adjuvantes. 
Tabela 5. Porcentagem de controle em pós-emergência de trapoeraba (Commelina benghalensis) aos 7,14 e 21 DAA, após dessecação de présemeadura com apenas uma única aplicação (1 aplicação) ou aplicação sequencial (2 aplicações). Campo Mourão/PR, 2010.

\begin{tabular}{|c|c|c|c|c|c|c|c|}
\hline \multirow{2}{*}{$\begin{array}{l}\text { TRATAMENTOS (Dessecação Pré-semeadura) } \\
\text { Aplicação única (40 DAS) / } \\
\text { Aplicação sequencial (25 DAS) }\end{array}$} & \multirow{2}{*}{$\frac{\text { Pós-emergência }}{(28 \text { DDS })}$} & \multicolumn{2}{|c|}{7 DAA } & \multicolumn{2}{|c|}{14 DAA } & \multicolumn{2}{|c|}{$21 \mathrm{DAA}$} \\
\hline & & Única & Sequencial & Única & Sequencial & Única & Sequencial \\
\hline $\begin{array}{l}\text { 1. glyph. Polaris }{ }^{\circledR / 1} / \\
\text { glyph. Agripec }\end{array}$ & metsulfuron $^{17}$ & 12,5 a $\mathrm{B}$ & 17,5 a $\mathrm{A}$ & $44,5 \mathrm{~d}$ & $50,8 \mathrm{~d}$ & $52,5 \quad \mathrm{c}$ & $55,0 \quad \mathrm{~b}$ \\
\hline $\begin{array}{l}\text { 2. glyph. Polaris }{ }^{\circledR / 1}+\text { Triunfo } 515^{\circledR / 8} / \\
\text { glyph. Agripec }{ }^{\circledR / 3}+2,4-D^{14}+\text { Triunfo } 515^{\circledast / 6}\end{array}$ & $\begin{array}{l}\text { metsulfuron }{ }^{17}+ \\
\text { Triunfo } 515^{\circledR / 12}\end{array}$ & 13,3 a $A$ & 15,0 a $A$ & $47,0 \mathrm{~cd}$ & 55,3 bcd & $51,3 \quad \mathrm{c}$ & 53,3 \\
\hline $\begin{array}{l}\text { 3. glyph. Polaris }{ }^{\circledR / 1}+\text { Grap Super Gun } \\
\text { glyph. Agripec }{ }^{\circledR / 3}+2,4-D^{1 / 4}+\text { Grap Sup Gun }\end{array}$ & $\begin{array}{l}\text { metsulfuron }^{17}+\text { Grap } \\
\text { Super Gun }\end{array}$ & 16,3 a $A$ & 19,5 a $\mathrm{A}$ & 48,8 bcd & 54,5 bcd & $61,5 \quad b c$ & 58,8 \\
\hline $\begin{array}{l}\text { 4. glyph. Polaris }{ }^{\circledR / 1}+\text { Assist }^{\circledR / 10} / \\
\text { glyph. Agripec } \\
\text { (ब/3 }+2,4-\mathrm{D}^{1 / 4}+\text { Assist }^{\circledR / 8}\end{array}$ & $\begin{array}{l}\text { metsulfuron } \\
\text { Assist }^{(1 / 14}\end{array}$ & 16,5 a $A$ & 18,3 a $\mathrm{A}$ & 47,5 bcd & $50,8 \quad \mathrm{~d}$ & $57,8 \quad \mathrm{c}$ & 56,5 \\
\hline $\begin{array}{l}\text { 5. glyph. Polaris }{ }^{\circledR / 1}+\text { Fulltec }^{\circledR / 11} / \\
\text { glyphosate Agripec } \\
\text { (1/3 }+2,4-\mathrm{D}^{14}+\text { Fulltec }^{\circledR / 9}\end{array}$ & $\begin{array}{l}\text { metsulfuron }^{17}+ \\
\text { Fulltec }^{\otimes / 15}\end{array}$ & 14,8 a $\mathrm{A}$ & 15,8 a $\mathrm{A}$ & $45,0 \mathrm{~d}$ & $52,3 \mathrm{~cd}$ & $54,5 \quad \mathrm{c}$ & 54,0 \\
\hline $\begin{array}{l}\text { 6. glyph. Atanor }{ }^{\mathbb{B} / 2} / \\
\text { glyph. Agripec }{ }^{\circledR / 3}+2,4-\mathrm{D}^{14}+\text { metsulfuron }^{16}\end{array}$ & $\begin{array}{l}\text { metsulfuron } \\
D^{15}+2,4-\end{array}$ & 14,5 a $A$ & 16,5 a $A$ & 60,0 abcd & $70,3 \mathrm{ab}$ & $75,0 \quad a b$ & 76,3 \\
\hline $\begin{array}{l}\text { 7. glyph. Atanor } \\
\text { glyph.Agripec }{ }^{\circledR / 3}+2,4-\mathrm{T}^{1 / 4}+\text { metsul }^{1 / 6}+{ }^{16} / \\
\text { Triunfo } 515^{\circledast / 6}\end{array}$ & $\begin{array}{l}\text { metsul. }^{/ 7}+2,4-\mathrm{D}^{15}+ \\
\text { Triunfo } 515^{\circledast / 12}\end{array}$ & 15,3 a $A$ & 18,3 a $\mathrm{A}$ & $65,3 \mathrm{ab}$ & 73,5 a & $85,0 \quad \mathrm{a}$ & 87,5 \\
\hline $\begin{array}{l}\text { 8. glyph. Atanor }{ }^{\otimes / 2}+\text { Grap Super Gun }^{\otimes / 9} / \\
\text { glyph.Agripec }{ }^{\otimes / 3}+2,4-D^{14}+\text { metsul. }^{/ 6}+\text { GrapS.Gun }^{\otimes / 7}\end{array}$ & $\begin{array}{l}\text { metsul. }^{17}+2,4-\mathrm{D}^{15}+ \\
\text { Grap Sup Gun }^{\circledR / 13}\end{array}$ & 16,5 a $A$ & 17,8 a $\mathrm{A}$ & 67,5 a & 74,8 а & 82,5 a & $85,0 \quad a$ \\
\hline 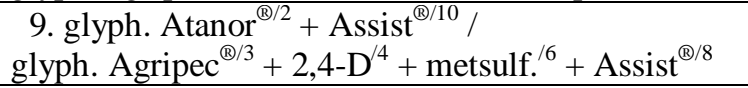 & $\underset{\text { Assist }^{\Theta / 14}}{\operatorname{metsul}} /{ }^{17}+2,4-\mathrm{D}^{15}+$ & 14,5 a $\mathrm{A}$ & 17,0 a $\mathrm{A}$ & $63,8 \mathrm{abc}$ & 69,8 abc & 80,3 a & 80,8 a \\
\hline $\begin{array}{l}\text { 10. glyph. Atanor } r^{\circledR / 2}+\text { Fulltec }^{\circledR / 11} / \\
\text { glyph. Agripec }{ }^{\otimes / 3}+2,4-D^{/ 4}+\text { metsul. }^{1 / 6}+\text { Fulltec }^{\circledast / 9}\end{array}$ & $\begin{array}{l}\text { metsul. }_{. / 17}+2,4-\mathrm{D}^{15}+ \\
\text { Fulltec }^{\otimes / 15}\end{array}$ & 13,8 a $\mathrm{B}$ & 18,3 a $\mathrm{A}$ & 60,8 abcd & 71,0 ab & 84,5 & 81,3 \\
\hline 11. testemunha sem aplicação & - & 0,0 b $\quad$ A & $0,0 \quad \mathrm{~b} \quad \mathrm{~A}$ & 0,0 ᄅ & $0,0 \mathrm{e}$ & $0,0 \quad \mathrm{~d}$ & $0,0 \quad \mathrm{c}$ \\
\hline \multicolumn{2}{|l|}{ Coeficiente de Variação } & \multicolumn{2}{|c|}{$\mathrm{CV}(\%)=21,45$} & \multicolumn{2}{|c|}{$\mathrm{CV}(\%)=13,53$} & \multicolumn{2}{|c|}{$\mathrm{CV}(\%)=11,07$} \\
\hline \multicolumn{2}{|l|}{ Teste f (F tratamentos e F aplicação) } & $\begin{array}{c}\text { Fcaltrat }=20,1 \\
*\end{array}$ & $\begin{array}{c}\mathrm{DMS}_{\text {trat }}=7,4 \\
0\end{array}$ & $\begin{array}{c}\text { Fcal } \\
\text { trat }=55,4^{*}\end{array}$ & $\begin{array}{c}\mathrm{DMS}_{\text {trat }}=17,8 \\
9\end{array}$ & \begin{tabular}{c|} 
Fcal \\
trat $=102,5^{*}$
\end{tabular} & \begin{tabular}{|c|}
$\mathrm{DMS}_{\text {trat }}=16,3$ \\
2
\end{tabular} \\
\hline \multicolumn{2}{|l|}{ DMS (5\%) } & \begin{tabular}{|c|} 
Fcalaplic $=12$, \\
$5^{*}$
\end{tabular} & $\mathrm{DMS}_{\text {Aplic }}=4,4$ & F aplic $=16,7^{\mathrm{NS}}$ & $\operatorname{DMS}_{\text {Aplic }}=10$ & F aplic $=0,05^{\mathrm{NS}}$ & $\operatorname{DMS}_{\text {Aplic }}=9,7$ \\
\hline
\end{tabular}


Outra vantagem da associação entre metsulfuron-methyl e 2,4-D, juntamente com adjuvantes alternativos, refere-se à possibilidade de controle de plantas daninhas resistentes aos herbicidas inibidores da enzima ALS, como é o caso do metsulfuron-methyl. Segundo Roman et al. (2006), na cultura do trigo já foram encontrados biótipos de Raphanus sativus (nabiça) resistentes à ação de metsulfuron-methyl e de outros herbicidas inibidores de ALS.

Quanto aos aspectos de fitointoxicação, foram constatados aos 7 e 14 DAA e 21/7 DAA apenas leves sintomas visuais de amarelecimento das bordas das folhas de algumas plantas, sendo estes em média, inferiores a 4\% (Tabela 6). Desta forma, os resultados não caracterizam ação fitotoxica à cultura do trigo para aplicação em pósemergência de metsulfuron-methyl e metsulfuron-methyl $+2,4-\mathrm{D}$, associados aos adjuvantes estudados. Segundo Rodrigues \& Almeida (2005) e Lorenzi et al. (2006), os herbicidas metsulfuron-methyl e 2,4-D são recomendados como seletivos para cultura do trigo, com eficiência reconhecida no controle em pós-emergência inicial de trapoeraba (C. benghalensis). Entretanto, Hartwig et al. (2008), mencionam redução no desenvolvimento inicial de alguns genótipos de trigo submetidos à dose comercial de metsulfuron-methyl, indicando existir variabilidade genética entre cultivares de trigo para tolerância a este herbicida.

Com relação à produtividade da cultura do trigo, não foram constatadas diferenças significativas entre os sistemas de manejo em aplicação única e seqüencial, assim como os resultados também não evidenciaram distinções significativas para o controle de trapoeraba em pós-emergência e as associações de adjuvantes estudadas. Apesar da combinação de manejo em aplicação única e sequencial, juntamente com o controle de pósemergência, terem proporcionado aumento médio da produtividade em 33,0\% (Tabela 7), em relação às testemunhas, a explicação mais coerente para a inconsistência dos resultados fundamenta-se na ocorrência de baixa disponibilidade hídrica em boa parte do ciclo da cultura (Tabela 2).

Os resultados, de forma geral, indicam que a utilização dos adjuvantes estudados em mistura em tanque com herbicidas de manejo de pré-semeadura e/ou mesmo com herbicidas na pós-emergência do trigo, podem apresentar comportamento distinto em relação às espécies de plantas daninhas e sobre outras variáveis relacionadas ao sistema produtivo. Evidenciam ainda, que nem sempre as melhorias do desempenho herbicidas resultaram em ganhos da cultura em questão, o que caracteriza ainda mais a necessidade da ampliação do desenvolvimento de pesquisas e divulgação de informação sobre a ação dos adjuvantes. 
Tabela 6. Fitointoxicação da cultura do trigo aos 7, 14, 21/7 e 28/14DAA dos tratamentos em pós-emergência, considerando as condições de uma única aplicação (1 aplicação) ou aplicação sequencial (2 aplicações) da dessecação. Campo Mourão/PR, 2010.

\begin{tabular}{|c|c|c|c|c|c|c|c|}
\hline \multirow{2}{*}{$\begin{array}{l}\text { TRATAMENTOS (Dessecação Pré-semeadura) } \\
\text { Aplicação única (40 DAS) / } \\
\text { Aplicação sequencial (25 DAS) }\end{array}$} & \multirow{2}{*}{$\frac{\text { Pós-emergência }}{(28 \text { DDS })}$} & \multirow{2}{*}{$\frac{7 \text { DAA }}{\text { Única }}$} & \multirow{2}{*}{$\frac{14 \text { DAA }}{\text { Única }}$} & \multicolumn{2}{|c|}{ 21/7 DAA } & \multicolumn{2}{|c|}{$28 / 14$ DAA } \\
\hline & & & & Única & Sequencial & Única & Sequencial \\
\hline $\begin{array}{l}\text { 1. glyph. Polaris } \\
\text { glyph. Agripec }{ }^{\circledR / 3}+2,4-\mathrm{D}^{/ 4}\end{array}$ & metsulfuron $^{17}$ & 3,5 & 1,5 & 0,0 & 2,3 & 0,0 & 0,0 \\
\hline $\begin{array}{l}\text { 2. glyph. Polaris }{ }^{\circledR / 1}+\text { Triunfo } 515^{\circledR / 8} / \\
\text { glyph. Agripec }{ }^{\circledR / 3}+2,4-D^{14}+\text { Triunfo } 515^{\circledast / 6}\end{array}$ & $\begin{array}{l}\text { metsulfuron }^{17}+ \\
\text { Triunfo } 515^{\circledR / 12}\end{array}$ & 3,5 & 2,3 & 0,0 & 3,0 & 0,0 & 0,0 \\
\hline 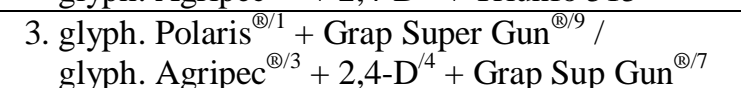 & $\begin{array}{l}\text { metsulfuron }^{17}+\text { Grap } \\
\text { Super Gun }\end{array}$ & 3,0 & 2,3 & 0,0 & 3,0 & 0,0 & 0,0 \\
\hline $\begin{array}{l}\text { 4. glyph. Polaris }{ }^{\Theta / 1}+\text { Assist }^{\circledR / 10} / \\
\text { glyph. Agripec } \\
\text { (I/3 }+2,4-\mathrm{D}^{1 / 4}+\text { Assist }^{\circledR / 8}\end{array}$ & $\begin{array}{l}\text { metsulfuron } \\
\text { Assist }^{\circledR / 14}+\end{array}$ & 3,0 & 3,3 & 0,0 & 2,8 & 0,0 & 0,0 \\
\hline $\begin{array}{l}\text { 5. glyph. Polaris }{ }^{\circledR / 1}+\text { Fulltec }^{\circledR / 11} / \\
\text { glyphosate Agripec }^{\circledR / 3}+2,4-\mathrm{D}^{/ 4}+\text { Fulltec }^{\circledR / 9}\end{array}$ & $\begin{array}{l}\text { metsulfuron }^{17}+ \\
\text { Fulltec }^{\circledR / 15}\end{array}$ & 3,5 & 2,8 & 0,0 & 3,3 & 0,0 & 0,0 \\
\hline 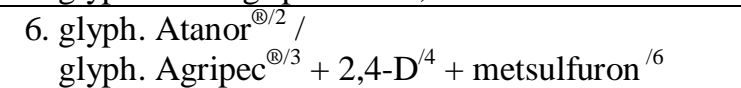 & $\begin{array}{l}\text { metsulfuron } \\
\mathrm{D}^{1 / 5}+2,4-\end{array}$ & 3,0 & 2,8 & 0,0 & 2,8 & 0,0 & 0,0 \\
\hline 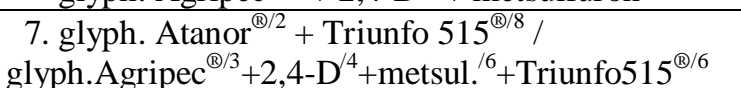 & $\begin{array}{l}\text { metsul. }^{17}+2,4-\mathrm{D}^{15}+ \\
\text { Triunfo } 515^{\circledR / 12}\end{array}$ & 3,5 & 2,0 & 0,0 & 2,8 & 0,0 & 0,0 \\
\hline $\begin{array}{l}\text { 8. glyph. Atanor }{ }^{\circledR / 2}+\text { Grap Super Gun }^{\circledR / 9} / \\
\text { glyph.Agripec }{ }^{\circledR / 3}+2,4-\mathrm{D}^{1 / 4}+\text { metsul. }^{/ 6}+\text { GrapS.Gun }^{\circledR / 7}\end{array}$ & $\begin{array}{l}\text { metsul. }^{17}+2,4-\mathrm{D}^{15}+ \\
\text { Grap Sup Gun }\end{array}$ & 3,5 & 1,5 & 0,0 & 3,0 & 0,0 & 0,0 \\
\hline 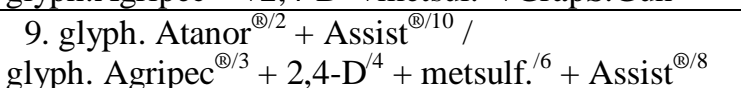 & 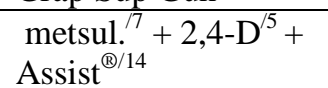 & 4,0 & 2,8 & 0,0 & 3,5 & 0,0 & 0,0 \\
\hline $\begin{array}{l}\text { 10. glyph. Atanor }{ }^{\circledR / 2}+\text { Fulltec }^{\circledR / 11} / \\
\text { glyph. Agripec }{ }^{\circledR / 3}+2,4-D^{14}+\text { metsul. }^{/ 6}+\text { Fulltec }^{\circledR / 9}\end{array}$ & $\begin{array}{l}\text { metsul. }_{.17}^{17}+2,4-D^{15}+ \\
\text { Fulltec }^{\circledR 1 / 15}\end{array}$ & 3,5 & 2,0 & 0,0 & 3,3 & 0,0 & 0,0 \\
\hline 11. testemunha sem aplicação & - & 0,0 & 0,0 & 0,0 & 0,0 & 0,0 & 0,0 \\
\hline
\end{tabular}

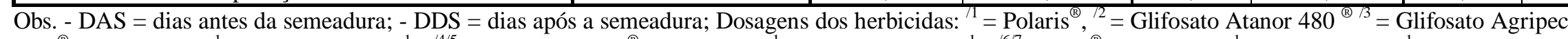

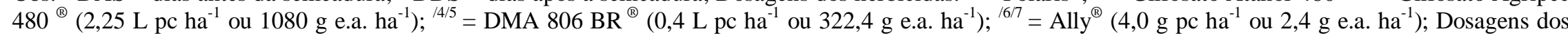
adjuvantes: ${ }^{1 / 9 / 11}=70 \mathrm{~mL} \mathrm{pc} \mathrm{ha}{ }^{-1} ;{ }^{/ 12 / 13 / 15}=80 \mathrm{~mL} \mathrm{pc} \mathrm{ha}^{-1} \mathrm{e}^{/ 10 / 14}=500 \mathrm{~mL} \mathrm{pc} \mathrm{ha-1}$. - Médias seguidas de mesma letra maiúscula na linha e minúscula na coluna não diferem entre si pelo teste Tukey a $5 \%$ de probabilidade. $*=\mathrm{P}<0,05 ;{ }^{\mathrm{NS}}=$ não significativo. 
Tabela 7. Produtividade da cultura do trigo $\left(\mathrm{kg} \mathrm{ha}^{-1}\right)$, considerando as condições de uma única aplicação ( 1 aplicação) ou aplicação sequencial (2 aplicações) da dessecação. Campo Mourão/PR, 2010.

\begin{tabular}{|c|c|c|c|}
\hline \multirow{2}{*}{$\begin{array}{l}\text { TRATAMENTOS (Dessecação Pré-semeadura) } \\
\text { Aplicação única (40 DAS) / } \\
\text { Aplicação sequencial (25 DAS) }\end{array}$} & Pós-emergência & \multicolumn{2}{|c|}{ Produtividade $\left(\mathrm{kg} \mathrm{ha}^{-1}\right)$} \\
\hline & (28 DDS) & Única & Sequencial \\
\hline $\begin{array}{l}\text { 1. glyphosate Polaris }{ }^{\circledR / 1} / \\
\text { glyphosate Agripec }^{\circledR / 3}+2,4-\mathrm{D}^{14}\end{array}$ & 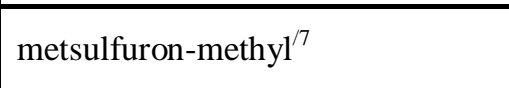 & $1766 \mathrm{ab}$ & $1645 \mathrm{bc}$ \\
\hline $\begin{array}{l}\text { 2. glyphosate Polaris } s^{\circledR / 1}+\text { Triunfo } 515^{\circledast / 8} / \\
\text { glyphosate Agripec }{ }^{\circledR / 3}+2,4-D^{14}+\text { Triunfo } 515^{\circledast / 6}\end{array}$ & 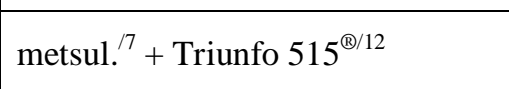 & 1852 a & 1809 \\
\hline $\begin{array}{l}\text { 3. glyphosate Polaris }{ }^{\circledR / 1}+\text { Grap Super Gun }^{\circledR / 9} / \\
\text { glyphosate Agripec }^{\circledR / 3}+2,4-\mathrm{D}^{14}+\text { Grap Super Gun }\end{array}$ & metsul. $^{17}+$ Grap Sup. Gun ${ }^{\circledR / 13}$ & $1788 \mathrm{ab}$ & 1747 \\
\hline $\begin{array}{l}\text { 4. glyphosate Polaris }{ }^{\circledast / 1}+\text { Assist }^{\circledR / 10} / \\
\text { glyphosate Agripec }^{\circledR / 3}+2,4-\mathrm{D}^{1 / 4}+\text { Assist }^{\circledR / 8}\end{array}$ & metsulfuron $^{17}+$ Assist $^{\circledR / 14}$ & $1794 \mathrm{ab}$ & 1748 \\
\hline $\begin{array}{l}\text { 5. glyphosate Polaris } \mathrm{s}^{\circledR / 1}+\text { Fulltec }^{\circledR / 11} / \\
\text { glyphosate Agripec } \\
\text { (l/3 } \\
+2,4-\mathrm{D}^{14}+\text { Fulltec }^{\circledR / 9}\end{array}$ & metsulfuron $^{17}+$ Fulltec $^{\circledR / 15}$ & 1822 a & 1695 \\
\hline $\begin{array}{l}\text { 6. glyphosate Atanor } \\
\text { glyphosate Agripec }^{\circledR / 3}+2,4-\mathrm{D}^{14}+\text { metsulfuron-methyl }^{16}\end{array}$ & metsulfuron $^{17}+2,4-\mathrm{D}^{15}$ & 2054 a & 1974 \\
\hline 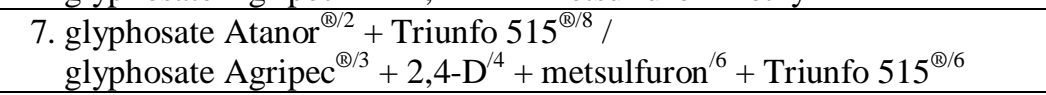 & metsul. $.^{17}+2,4-\mathrm{D}^{15}+$ Triunfo $515^{\circledast / 12}$ & 2186 a & 2161 \\
\hline $\begin{array}{l}\text { 8. glyphosate Atanor } \\
\text { glyphosate Agripec }{ }^{\circledR / 3}+2,4-\mathrm{D}^{1 / 4}+\text { metsulfuron }^{16}+\text { Grap Super Gun }^{\circledR / 7}\end{array}$ & metsul. $^{17}+2,4-\mathrm{D}^{15}+$ Grap S. Gun ${ }^{\circledR / 13}$ & 2226 a & $2012 \mathrm{ab}$ \\
\hline $\begin{array}{l}\text { 9. glyphosate Atanor }{ }^{\circledR / 2}+\text { Assist }^{\circledR / 10} / \\
\text { glyphosate Agripec }^{\circledR / 3}+2,4-\mathrm{D}^{14}+\text { metsulfuron }^{16}+\text { Assist }^{\circledR / 8}\end{array}$ & metsul. $^{17}+2,4-\mathrm{D}^{15}+$ Assist $^{\circledR / 14}$ & 2050 a & 1927 \\
\hline $\begin{array}{l}\text { 10. glyphosate Atanor }{ }^{\circledR / 2}+\text { Fullte }^{\circledR / 11} / \\
\text { glyphosate Agripec }^{\circledR / 3}+2,4-\mathrm{D}^{/ 4}+\text { metsulfuron }^{/ 6}+\text { Fulltec }^{\circledast / 9}\end{array}$ & metsul. $^{17}+2,4-D^{15}+$ Fulltec $^{\circledR / 15}$ & 2124 & 2105 \\
\hline 11. testemunha sem aplicação & - & 1316 & 1258 \\
\hline \multicolumn{2}{|l|}{ Coeficiente de Variação } & \multicolumn{2}{|c|}{$\mathrm{CV}(\%)=11,34$} \\
\hline \multicolumn{2}{|l|}{ Teste $\mathrm{f}$ ( $\mathrm{F}$ tratamentos e $\mathrm{F}$ aplicação) } & Fcal trat $=11,6^{*}$ & Fcal aplic $=3,27^{\mathrm{NS}}$ \\
\hline \multicolumn{2}{|l|}{ DMS (5\%) } & $\mathrm{DMS}_{\text {trat }}=499,71$ & DMS $_{\text {Aplic }}=298,95$ \\
\hline
\end{tabular}

Obs. - DAS = dias antes da semeadura; - DDS = dias após a semeadura; Dosagens dos herbicidas: ${ }^{11}=$ Polaris ${ }^{\circledR},{ }^{12}=$ Glifosato Atanor $480{ }^{\circledR / 3}=$ Glifosato Agripec

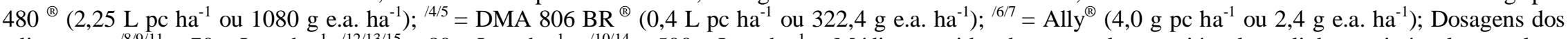
adjuvantes: ${ }^{18 / 9 / 11}=70 \mathrm{~mL} \mathrm{pc} \mathrm{ha}{ }^{-1} ;{ }^{112 / 13 / 15}=80 \mathrm{~mL} \mathrm{pc} \mathrm{ha}^{-1} \mathrm{e}^{/ 10 / 14}=500 \mathrm{~mL} \mathrm{pc} \mathrm{ha}^{-1}$. - Médias seguidas de mesma letra maiúscula na linha e minúscula na coluna não diferem entre si pelo teste Tukey a $5 \%$ de probabilidade. $*=\mathrm{P}<0,05 ;{ }^{\mathrm{NS}}=$ não significativo. . 


\section{Conclusões}

Os adjuvantes Triunfo $515^{\circledR}$, Grap Super Gun $^{\circledR}$ e $\operatorname{Assist}^{\circledR}\left(70 \mathrm{~mL}\right.$ pc ha $\left.{ }^{-1}\right)$ promovem incremento no controle de capimamargoso (Digitaria insularis) para as formulações de glyphosate Polaris ${ }^{\circledR}$ e Atanor $480^{\circledR}\left(2,25 \mathrm{~L} \mathrm{pc} \mathrm{ha}^{-1}\right)$, em aplicação única de pré-semeadura;

Para trapoeraba (Commelina benghalensis), apesar da baixa eficiência da dessecação de pré-semeadura, a utilização dos adjuvantes incrementa a ação do glyphosate isoladas e/ou em mistura com 2,4-D e 2,4-D + metsulfuron-methyl;

As associações entre metsulfuronmethyl + 2,4-D, com os adjuvantes, controla satisfatoriamente a trapoeraba em pósemergência.

Metsulfuron-methyl $\left(2,4\right.$ g e.a. ha $\left.{ }^{-1}\right)$ e metsulfuron-methyl + 2,4-D $\left(2,4 \mathrm{~g}\right.$ e.a. $\left.\mathrm{ha}^{-1}\right)$, associados aos adjuvantes Triunfo $515^{\circledR}$, Grap Super Gun ${ }^{\circledR}$, Assist $^{\circledR}$ e Fulltec ${ }^{\circledR}$ não promove ação fitotoxica prejudicial à cultura do trigo $\mathrm{cv}$ BRS 220.

\section{Referências}

AGOSTINeTtO, D. et al. Período crítico de competição de plantas daninhas com a cultura do trigo. Planta Daninha, v.26, n.2, p.271278, 2008.

ALBRECHT, A.J.P. et al. Metsulfuron-methyl no desempenho agronômico e na qualidade das sementes de trigo. Revista Brasileira de Herbicidas, v.9, n.2, p.54-62, 2010.

AMBROSI, I. et al. Aspectos econômicos da cadeia produtiva de trigo no Brasil. Passo Fundo: Embrapa Trigo, 2000. 108p. (Embrapa Trigo. Documentos, 15).

COMISSÃO SUL-BRASILEIRA DE PESQUISA DE TRIGO. Indicações Técnicas da Comissão Sul-Brasileira de Pesquisa de Trigo e Triticale. Passo Fundo: EmbrapaCNPT, 2005. $234 \mathrm{p}$.

CONAB - COMPANHIA NACIONAL DE ABASTECIMENTO. Acompanhamento da Safra Brasileira de Grãos 2010/2011. Terceiro Levantamento. 2010. Disponível em: http://www.conab.gov.br/OlalaCMS/uploads/ar quivos/10_12_09_16_39_39_boletim_portugue s_-dez_de_2010.pdf> Acesso em: 15 dez. 2010.

DAN, H.A. et al. Adjuvantes multifuncionais associados ao herbicida glyphosate no controle de Digitaria insularis. Global Science and Technology, v.3, n.2, p.30-38, 2010.

EL-DASH, A; MIRANDA, M.Z. Farinha integral de trigo germinado. Características nutricionais e estabilidade ao armazenamento. Ciência e Tecnologia dos Alimentos, v.22, n.3, p.216-223, 2002.

FERREIRA, R.A. Trigo: O alimento mais produzido no mundo. Nutrição Brasil, v.2, n.1, p.45-52, 2003.

GIECO, E.A.; DUBKOVSKY, J.; CAMARGO, L.E.A. Interaction between resistance to Septoria tritici and phonological stages in wheat. Scientia Agrícola, v.61, n.4, p.422-426, 2004.

GUTKOSKI, L.C.; JACOBSEN NETO, R. Procedimento para teste laboratorial de panificação - Pão tipo forma. Ciência Rural, v.32, n.5, p.873-879, 2002.

HARTWIG, I. et al. Tolerância de trigo (Triticum aestivum) e aveia (Avena sp.) a herbicidas inibidores da enzima acetolactato sintase (ALS). Planta Daninha, v.26, n.2, p.361-368, 2008.

IAPAR - INSTITUTO AGRONÕMICO DO PARANÁ. Informações técnicas para a cultura do trigo no Paraná - 2002. Londrina: IAPAR, 2002. 181p. (IAPAR. Circular, 122) 
LORENZI, H. et al. Manual de identificação e controle de plantas daninhas: plantio direto e convencional. $6^{\underline{a}}$ Ed., Nova Odessa, SP: Plantarum, 2006. 339p.

MACIEL, C.D.G. et al. Eficiência e qualidade da aplicação de glyphosate + carfentrazone no controle de Commelina diffusa em função da ponta de pulverização e ação do adjuvante Triunfo Flex ${ }^{\circledR}$. In: III SIMPÓSIO INTERNACIONAL SOBRE GLYPHOSATE, 2011, Botucatu. Anais... Botucatu: FEPAF, 2011. p.388-391.

PROCÓPIO, S.O. et al. Efeitos de dessecantes no controle de plantas daninhas na cultura da soja. Planta Daninha, v.24, n.1, p.193-197, 2006.

RODRIGUES, B.N.; ALMEIDA, F.S. Guia de herbicidas. 5- ed., Londrina: Edição dos Autores, 2005. 592p.

ROMAN, E.S.; VARGAS, L.; RODRIGUES, $O$. Manejo e controle de plantas daninhas em trigo. Passo Fundo: Embrapa Trigo, 2006. 12p. (Embrapa Trigo. Documentos Online, 63) Acesso: 13/06/2011. Disponível em: $<$ http://www.cnpt.embrapa.br/ biblio/do/p_do63.htm>

SALVAGIOTTI, F. et al. Efecto de la sobredosis de metsulfurón metil sobre el cultivo de trigo con diferentes niveles de fertilidad. Revista Para Mejorar La Producción, v.16, n.3, p.53-55, 2001.

SBCPD. Procedimentos para instalação, avaliação e análise de experimentos com herbicidas. Londrina: SBCPD, 1995, 42p.

VARGAS, L.; ROMAN, E.S. Seletividade e eficiência de herbicidas em cereais de inverno. Revista Brasileira de Herbicidas, v.3, n.1, p.1-10, 2005.

VELLOSO, J.A.R.O.; DAL'PIAZ, R. Controle de azevém (Lolium multiflorum L.) e gorga (Spergula arventis L.) e seletividade de herbicidas as culturas do trigo, cevada e centeio. Planta Daninha, v.5, n.2, p.8-13, 1982. 